\title{
Differential Spatial Frequency Modulations on Facial Emotion Detection and Perception
}

\author{
Ryan McBain ${ }^{1}$, Daniel Norton ${ }^{1}$ and Yue Chen ${ }^{*}, 1,2$ \\ ${ }^{I}$ Mailman Research Center, McLean Hospital, 115 Mill St. Belmont, MA 02478, USA \\ ${ }^{2}$ Department of Psychiatry, Harvard Medical School, 115 Mill St. Belmont, MA 02478, USA
}

\begin{abstract}
The recognition of facial affect is a core component of social cognition and is influenced by bottom-up and topdown processes. Spatial frequency-specific processing is a fundamental mechanism underpinning visual perception that also contributes to emotion processing. Yet, it remains relatively unclear how this bottom-up process modulates analysis of affect signals. In this study, face images were manipulated via high-pass and low-pass spatial frequency filtering, and the effects of these manipulations were measured on two tasks: emotion detection and emotion perception. The detection task measured individuals' ability to detect the presence of happiness or fear when relying only upon high spatial frequency (HSF), low spatial frequency (LSF) or unfiltered (BSF) images. The perception task evaluated individuals' tendency to perceive one type of images (e.g. LSF) as happier or more fearful than another (e.g. HSF). On the detection task, individuals were better at detecting happiness in LSF images as compared to HSF images, a result not found in the fear detection condition. On the perception task, images with HSFs were perceived as significantly happier than images without HSFs, but only at low emotional salience levels. No significant effect for spatial frequency manipulation was found in the fear perception condition. Together, these results highlight the significant bottom-up role of spatial frequency content in perceiving happiness as opposed to fear and indicates the unique roles of bottom-up visual mechanisms when processing these two emotions.
\end{abstract}

Keywords: Face recognition, affect, vision, low spatial frequency, high spatial frequency.

\section{INTRODUCTION}

The recognition of facial affect plays a foundational role in our interpersonal interactions [1]. This social cognitive ability requires the application of contextual resources (topdown) while processing the visual information presented (bottom-up). It has been shown that cortical response to fearful face stimuli is associated with task relevance [2] and the covertness of the faces presented [3], as such emphasizing the importance of top-down factors in affect perception. Yet, other evidence indicates that amygdala activation in response to fearful faces occurs independent of visual attention $[4,5]$, thus indicating the role of more automatic, lower-level processes. Taken together, findings like these underscore the complex nature of facial emotion recognition and highlight the need for new and innovative approaches for assessing bottom-up and top-down mechanisms.

The brain processes visual images, including facial images, based upon functional units that are tuned to specific spatial frequency bands $[6,7]$. Some units are tuned to low spatial frequencies (broad, configural information), and some to high spatial frequencies (fine-detail) [8]. This aspect of the early visual system may offer a unique vantage point for studying bottom-up processing of facial emotion recognition.

*Address correspondence to this author at the McLean Hospital, Department of Psychiatry, Harvard Medical School, 115 Mill St. Belmont, MA 02478, USA; Tel: 617855 3615; Fax: 6178553611 ;

E-mails: ychen@mclean.harvard.edu,yur_chen_online@yahoo.com
The reasons for this are twofold: First, brain regions associated with face-specific (e.g., fusiform gyrus) and affective (e.g., amygdala) processing are sensitive to distinct SF bands. For example, face images composed of high spatial frequencies (HSFs) activate the left fusiform gyrus and inferior occipital gyrus more than those composed of low spatial frequencies (LSFs) [9], while the bilateral middle occipital gyrus responds more to manipulations of LSFs [10]. Affective processing areas such as the amygdala have also been shown to differentially respond to LSFs of negative-valence images $[11,12]$. This dissociation is important because it indicates that manipulations in SF content can allow for inference as to the cortical mechanisms involved.

Second, preliminary evidence suggests that SF content may influence perception of facial affect. Schyns and Oliva found that the SF scale utilized for one facial categorization task (e.g., facial identity discrimination) can transfer to resolve a different categorization task (e.g., facial emotion recognition), indicating that emotion categorization is driven in part by SF processing [8]. In a more recent study, Vuilleumier and colleagues found that individuals perceived facial images with HSFs as more fearful than those which only contained LSFs [12]. A third study, by White and Li, found that individuals were slower at matching emotions of LSF facial images than BSF images, whereas matching facial identities was unaffected by SF manipulation [13]. While these findings are intriguing, numerous questions remain. All three studies utilized images only at a high level of emotion salience, leaving unanswered the question of how 
SF content and perception of emotional salience are related. The study by Vuilleumier and colleagues only assessed perception of fearfulness, raising the question whether modulation of SF content affects perception of other emotions [12]; and the study by White and Li only found differences in reaction time [13], leaving open the question of whether other performance measures like accuracy are affected. A systematic analysis of the role of SF information in facial affect recognition is required to resolve such questions.

The present study utilized a psychophysical approach for assessing individuals' abilities to detect and perceive facial affect in face images with modulated spatial frequency information. We systematically varied the emotional salience of facial images, and measured the degree of emotionality required for individuals to reliably detect fearfulness and happiness in BSF, HSF, and LSF face images (detection task). We then examined individuals' tendencies to attribute greater emotional salience to HSF, LSF, and BSF images (perception task). In conjunction, these two tasks allowed us to examine individuals' perceptual capacity to detect emotional content alongside their subjective tendency to perceive emotionality in certain image types. In both cases spatial frequency content was manipulated in order to observe the bottom-up effects of lower level processing on facial emotion recognition.

\section{EXPERIMENT 1: FACE EMOTION DETECTION}

\subsection{Methods}

\subsubsection{Subjects}

Twenty-nine subjects (14 females, 15 males; mean age $=$ 44.0 years, standard deviation 14.7 years) participated in the experiment. Inclusion criteria were defined as [1] aged between 18-65 years [2], verbal IQ greater than 70, as measured by the WAIS-R [3, 14] visual acuity of at least $20 / 30$, as measured by the Rosenbaum pocket vision screener [4], no drug or alcohol abuse within the past six months, and [5] no history of neurological or psychiatric disease. Criteria 4-5 were assessed during a standardized interview using the SCID-N/P [15].

\subsubsection{Procedures}

Targets for emotion detection were $6 \times 8.5 \mathrm{~cm}$ face images generated from the NimStim Face Stimulus Set [16]. These images contained either happy or fearful expressions of varying salience levels, created by morphing a neutral face $(0 \%)$ along a continuum with a highly emotive face $(100 \%)$ of the same individual. Morphing was done using FantaMorphPro 1.0 (2007). Six salience levels were used: 0, 6, 12, 24, 48 and $100 \%$.

Spatial frequency content of facial images was also systematically varied. Using Adobe Photoshop 5.0 (1998), three sets of stimuli were created: HSF filtered images, LSF filtered images, and BSF (unfiltered) images. The estimated cut-off value was 13.5 cycles per face width for low-pass filtering and 41.9 cycles per face width for high-pass filtering. Each face image was presented for $400 \mathrm{~ms}$, with a $500 \mathrm{~ms}$ inter-stimulus interval. Subjects performed the task in a darkened room, seated at a distance of $63 \mathrm{~cm}$ from the monitor.
For the emotion detection task, subjects were asked to determine which of two successively presented face images (one being a neutral face, the other being a face with varying levels of emotional salience) looked happier or more fearful (Fig. 1). The presentation of face images was blocked so that a given session contained only one emotion type (e.g., happiness) and one spatial frequency filtration (e.g., HSF images). In each session, 8 trials were repeated for each of the 6 comparison emotion salience levels $(0,6,12,24,48$, or $100 \%$ ). There were 2 presentation orders - the neutral face was presented first and the emotive face was presented second, or vice versa. Thus, the total number of trials was 96 per session. There were 6 testing sessions in total: 2 emotions (fear $v s$. happiness) x $3 \mathrm{SF}$ manipulations (HSF $v s$. LSF vs. BSF). Sessions were presented in a quasi-random order across subjects.

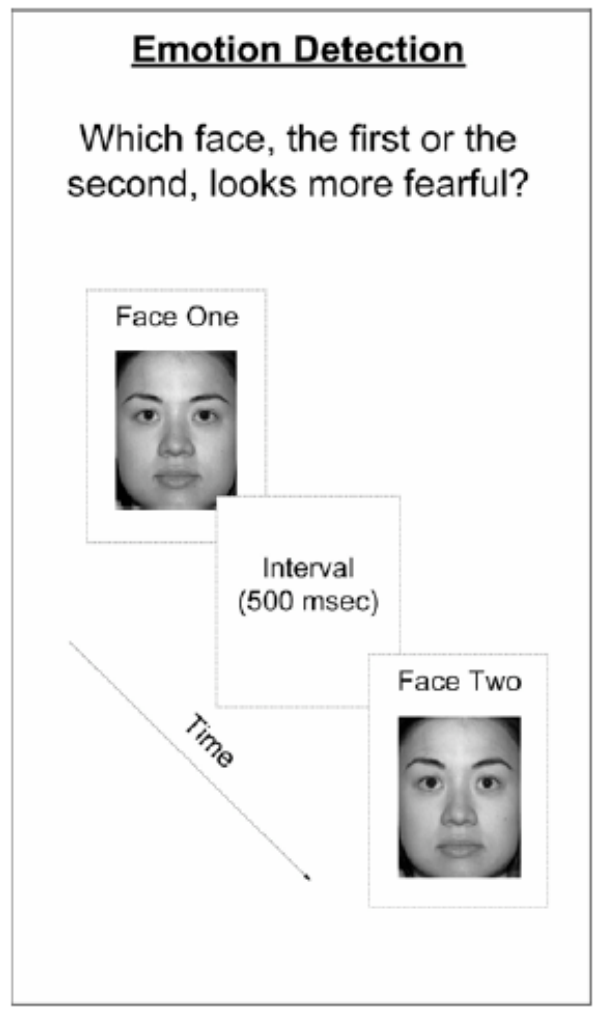

Fig. (1). Schematic illustration of the emotion detection task. The images presented in a given trial contain the same spatial frequency content (e.g., broad-band spatial frequency (BSF)), but differ in emotional salience (e.g., $0 \%$ vs. $15 \%$ ).

The critical performance measure was the minimal difference between the emotional and neutral faces at which subjects performed at the criterion of $80 \%$ correct. This measure can be extracted from a psychometric function (Weibull function) of the percent correct scores, and is defined as the perceptual threshold for detecting the designated stimulus attributes. The threshold was determined for each type of emotion (happiness and fear) and for each SF manipulation (LSF, HSF and BSF images). The logarithm of each perceptual threshold was then computed in order to arrive at subjects' detection scores.

The protocol was approved by the institutional review board of McLean Hospital. Written informed consent was obtained from each subject prior to participation. 
a

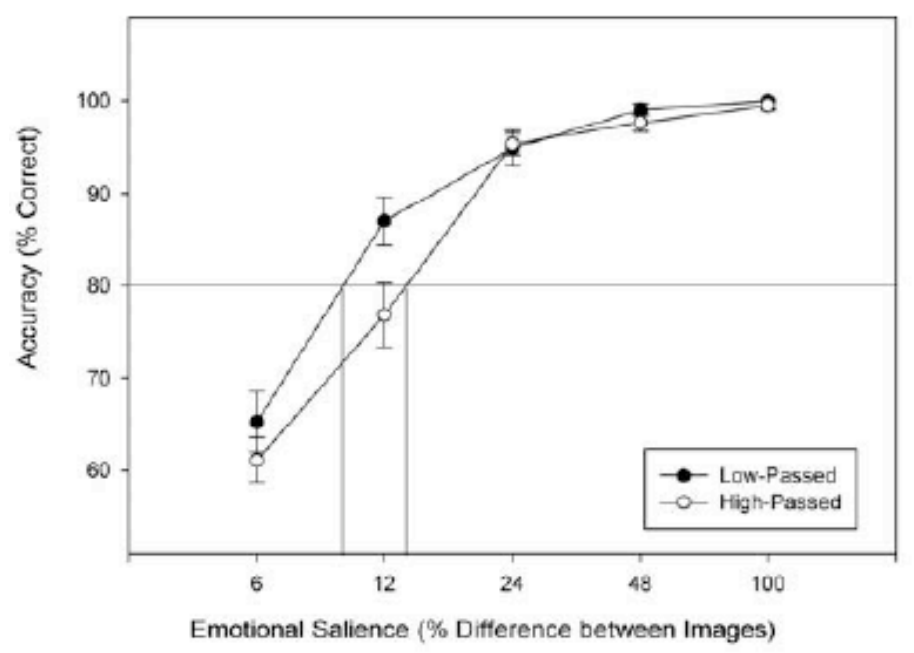

b

Fear Detection

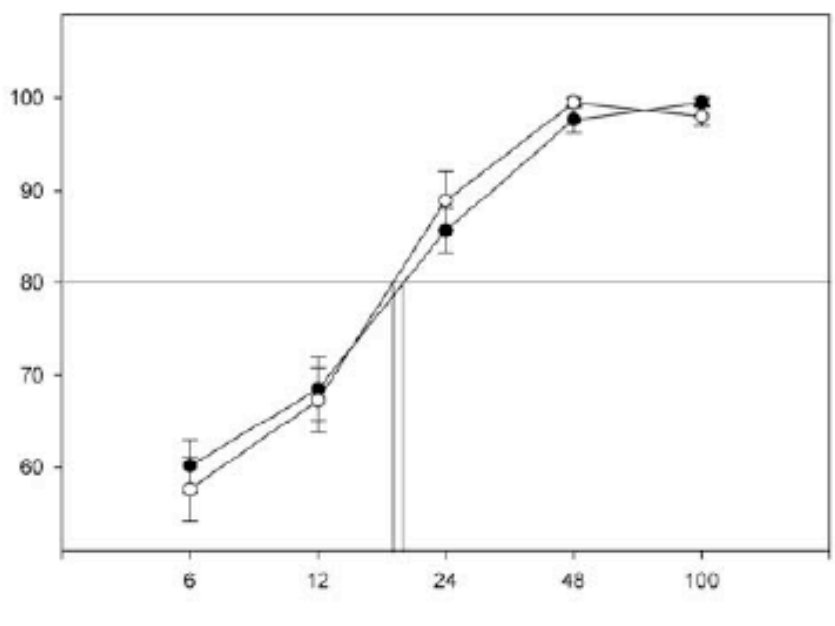

Fig. (2). (a) Performance on the fear detection task. The data acquired from the LSF and HSF images were averaged across subjects. The mean thresholds for the low- and high-passed images derived here (Exp. 1) were useful for setting up threshold levels of emotion salience, at which emotion perception of the images with different spatial frequency contents could be compared (Exp. 2). The $\mathrm{x}$-axis specifies the difference in emotional salience between facial images; the y-axis is percent correct for subjects' performance. The horizontal line denotes the $80 \%$ accuracy level, and the vertical lines mark the stimulus level needed for $80 \%$ accuracy on each SF condition (the threshold). (b) Average performance across subjects on the LSF and HSF conditions of the happiness detection task. In both panels, the error bars indicate \pm 1 standard error.

\subsection{Results and Discussion}

\subsubsection{Happiness Detection}

An ANOVA was used to compare individuals' detection scores across SF filtration types (LSF vs. HSF, HSF vs. BSF, LSF $v s$. BSF); the main effect was non-significant $\left(F_{2,84}\right.$ $=1.85, M S E=0.03, p>0.05)$. However, post hoc two-sample $\mathrm{t}$ tests revealed that detection scores were significantly lower (better performance) for low-passed images than for highpassed images $\left(t_{56}=3.02, p<0.01, \delta=0.43\right)$ (see Fig. 2). Other comparisons were non-significant ( $\mathrm{p}>0.05)$.

\subsubsection{Fear Detection}

An ANOVA was again used to compare individuals' detection scores across the different spatial frequency filtration types. No main effect was found $\left(F_{2,84}=0.19, M S E=\right.$ $0.04, p>0.05$ ). Post hoc t-tests were also used to compare individual SF manipulations (high-passed vs. low-passed, high-passed $v s$. unfiltered, low-passed $v s$. unfiltered). None of these comparisons were significant $(p>0.05)$, indicating that HSFs and LSFs play similar roles in their ability to detect fear.

\subsubsection{Discussion}

These results show that LSF content played a significantly greater role than HSF content in individuals' ability to detect happiness. However, manipulation of LSF and HSF content did not affect performance on the fear detection task. These contrasting results suggest that the brain mechanisms underpinning detection of fear and happiness differ in their use of bottom-up visual information. Happiness detection in particular appears to rely more on coarse, configural information, since subjects were less capable of detecting happiness in images lacking these inputs. This finding is particularly important since previous studies have mainly focused on the relationship between fear processing and LSFs, leaving unanswered the relationship between SF information and other emotions like happiness.

\section{EXPERIMENT 2: FACIAL EMOTION PERCEP- TION AS A FUNCTION OF SPATIAL FREQUENT CONTENT}

\subsection{Methods}

\subsubsection{Subjects}

Twenty-seven subjects from Experiment 1 participated in Experiment 2.

\subsubsection{Procedures}

Targets for emotion perception were the same as in Experiment 1 . However, for this experiment only 3 levels of emotional salience were utilized: $0 \%$ (neutral expression), threshold level (the minimum emotional salience level at which a given subject could detect an emotional expression with $80 \%$ accuracy, as derived from Experiment 1), and $100 \%$ (highly emotional expression).

The task was to determine which of two successively presented faces looked happier or more fearful (see Fig. 3). For each comparison in a trial, the two images were the same emotional salience $(0 \%$, threshold level, or $100 \%)$ but differed in terms of SF filtration (BSF $v s$. LSF, BSF $v s$. HSF, HSF vs. LSF). This paradigm thus allows an evaluation of subjects' tendency to ascribe greater emotional salience to images containing different spatial frequency contents. Presentation of the face images was blocked so that a given session contained only one emotion type (happiness or fear). In each testing session, 8 trials were repeated for the 3 emotion salience levels and $3 \mathrm{SF}$ filtration comparisons 
(HSF $v s$. LSF, HSF $v s$. BSF, LSF $v s$. BSF), for a total of 72 trials. Faces were presented for $400 \mathrm{~ms}$, with a $500 \mathrm{~ms}$ interstimulus interval.

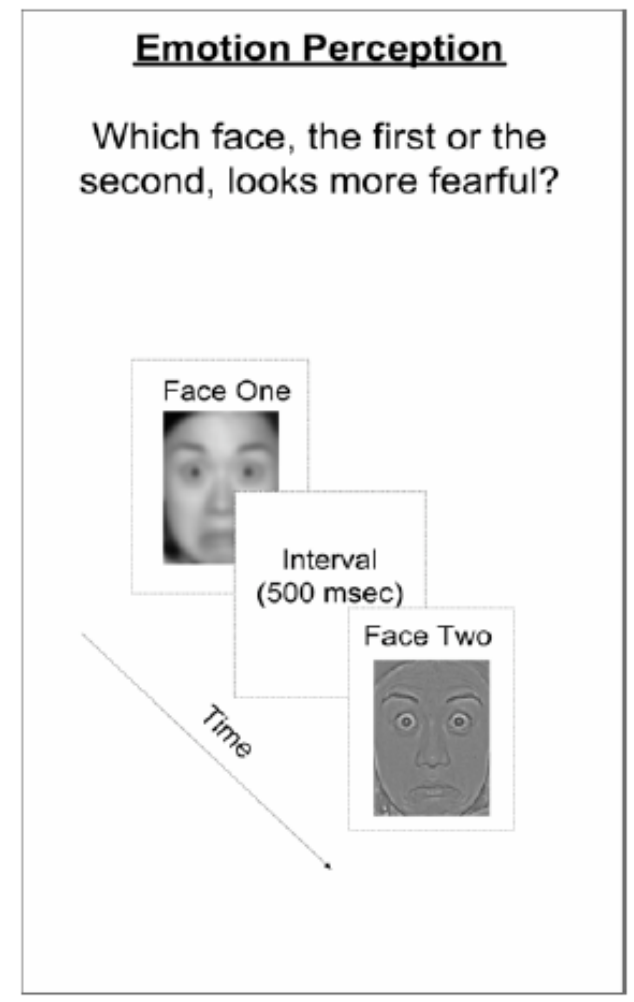

Fig. (3). Schematic illustration of the emotion perception task. The images presented in a given trial have the same emotional salience (e.g., 100\%), but differ in SF content (e.g., LSF vs. HSF).

The critical performance measure here was the proportion of comparison trials in which subjects chose images with LSFs (LSF and BSF) or HSFs (HSF and BSF) as happier or more fearful, as compared with those without LSFs (HSF) or without HSFs (LSF). By comparing images with vs. without a specified range of spatial frequencies, one could infer how LSFs and HSFs contributed to emotion perception in general, rather than merely examining the relative value of each. This measurement applied a two alternative forced-choice method to the direct comparison of individuals' perception of emotional content conveyed by differing SF bands.

\subsection{Results and Discussion}

\subsubsection{With vs. Without High Spatial Frequencies (HSFs)}

Two-way ANOVAs (SF manipulation $\mathrm{x}$ emotion salience) were conducted to inspect the effects of HSFs on each emotion type. On fear perception, there were no significant main effects or interactions $(p>0.05)$. On happiness perception, there was a significant main effect for SF manipulation $\left(F_{1,52}=48.63, M S E=0.059, p<0.001\right)$ : Images with HSFs were perceived as happier than those without HSFs. There was also a significant interaction effect between SF manipulation and salience $\left(F_{2,104}=12.60, p<0.001\right)$. To further inspect this interaction, paired t-tests were used to compare happiness perception at each salience level. As shown in Fig. (4), HSF information had a greater effect on happiness perception when stimuli were neutral $\left(t_{26}=4.63\right.$, $p<0.001, \delta=0.77)$ or at threshold level salience $\left(t_{26}=4.60\right.$, $p<0.001, \delta=0.75$ ), as compared to high emotional salience $(100 \%)$.

\subsubsection{With vs. Without Low Spatial Frequencies (LSFs)}

Again, two-way ANOVAs (SF manipulation $\mathrm{x}$ emotion salience) were used to assess the effects of LSF information on perception of each emotion type. On fear perception, there were no significant main or interaction effects. On happiness perception, there was a significant main effect for SF filtration $\left(F_{1,52}=31.98, M S E=0.033, p<0.001\right)$, signifying that images with LSFs were perceived as less happy. There was also an interaction between SF filtration and emotion salience $\left(F_{2,104}=20.78, p<0.001\right)$. Paired t-tests compared

Images with vs. Images without LSFs

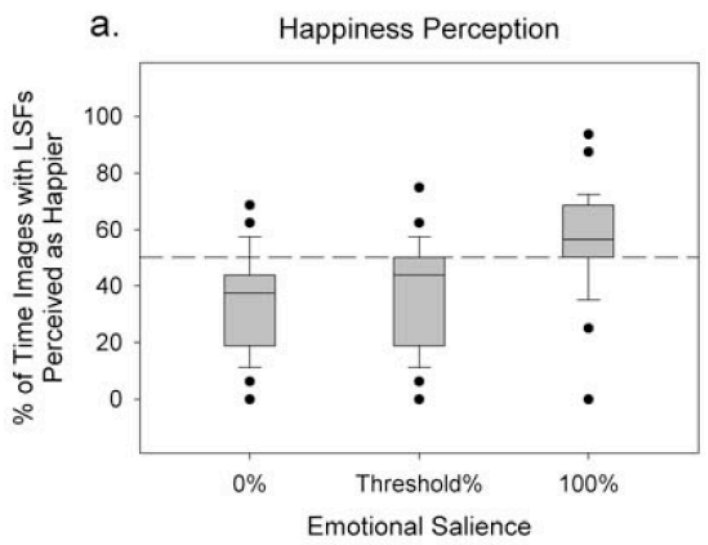

b.

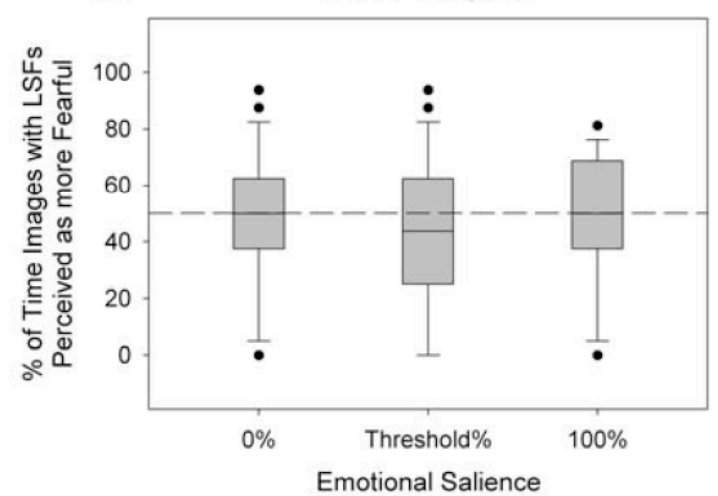

Fig. (4). (a) Box plot comparison of performance obtained from images with and without HSFs on the happiness perception task. The x-axis denotes emotional salience of the images being compared, while the y-axis represents the percent of the time images with HSFs were perceived as happier than images without HSFs. The dashed horizontal line shows chance level performance (50\%). (b) Box plot comparison of performance obtained from images with and without HSFs on the fear perception task. The x-axis denotes emotional salience of the images being compared, while the y-axis represents the percent of the time images with HSFs were perceived as more fearful than images without HSFs. 
images with and without LSFs at each salience level. Contrasted with highly emotive images, LSF information had a greater effect on happiness perception at non-emotive $\left(t_{26}=\right.$ $3.08, p=0.005, \delta=1.15)$ and threshold levels $\left(t_{26}=3.27, p=\right.$ $0.003, \delta=0.97$ ), as compared to high emotional salience (see Fig. 5).

\section{Facial Emotion Stimuli}

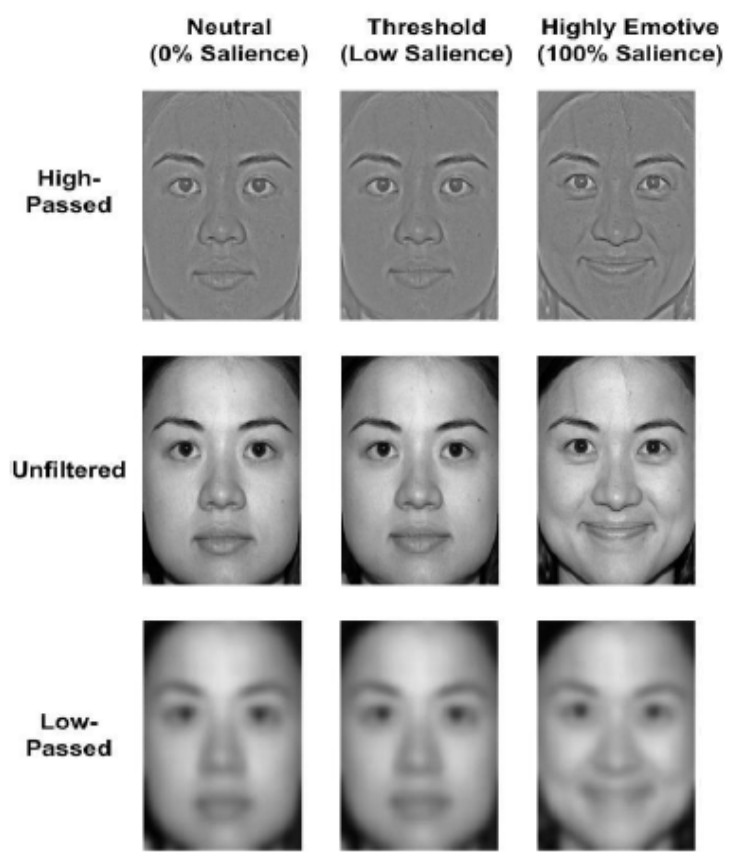

Fig. (5). (a) Box plot comparison of performance obtained from images with and without LSFs on the happiness perception task. The $\mathrm{x}$-axis denotes emotional salience of the images being compared, while the y-axis represents the percent of the time images with LSFs were perceived as happier than images without LSFs. The dashed horizontal line shows chance level performance $(50 \%)$. (b) Box plot comparison of performance obtained from images with and without LSFs on the fear perception task. The xaxis denotes emotional salience of the images being compared, while the $y$-axis represents the percent of the time images with LSFs were perceived as more fearful than images without LSFs.

\subsubsection{Discussion}

Modulation of SF information affected individuals' perception of happiness at low levels of emotional salience; however, SF modulations had no significant effect on fear perception. It is particularly noteworthy that HSF and LSF manipulations yielded opposite effects on happiness perception: At non- and low-emotive levels, HSF information facilitated the perception of happiness, while LSF information reduced the frequency of this percept.

Given the relatively weak relationship between SF filtration and fear perception, it may be suggested that happiness perception relies more upon the analysis of SF content, whereas fear perception is less influenced by this information. Consistent with these suggestions, a previous study using "Bubbles technique" method [17] found weak correlations between the spatial frequency filtering functions underpinning recognition of different emotions (e.g., sadness and anger). In a similar vein, this study shows that the utilization of spatial frequency information may be differentially involved in some emotions (like happiness) as opposed to others (like fear).

\section{GENERAL DISCUSSION}

\subsection{Happiness vs. Fear Perception}

In both Experiments, manipulations of SF content had a greater modulatory effect on individuals' recognition of happiness, as compared to their recognition of fear. On the detection task, happiness was easier to detect in LSF than HSF images (Fig. 6. bottom row, middle vs. top row, middle), while on the perception task images with HSFs were perceived as happier than those without HSFs (Fig. 6. top and middle rows $v s$. bottom row). On neither task did SF manipulations significantly affect individuals' ability to process fear information.

\section{Facial Emotion Stimuli}

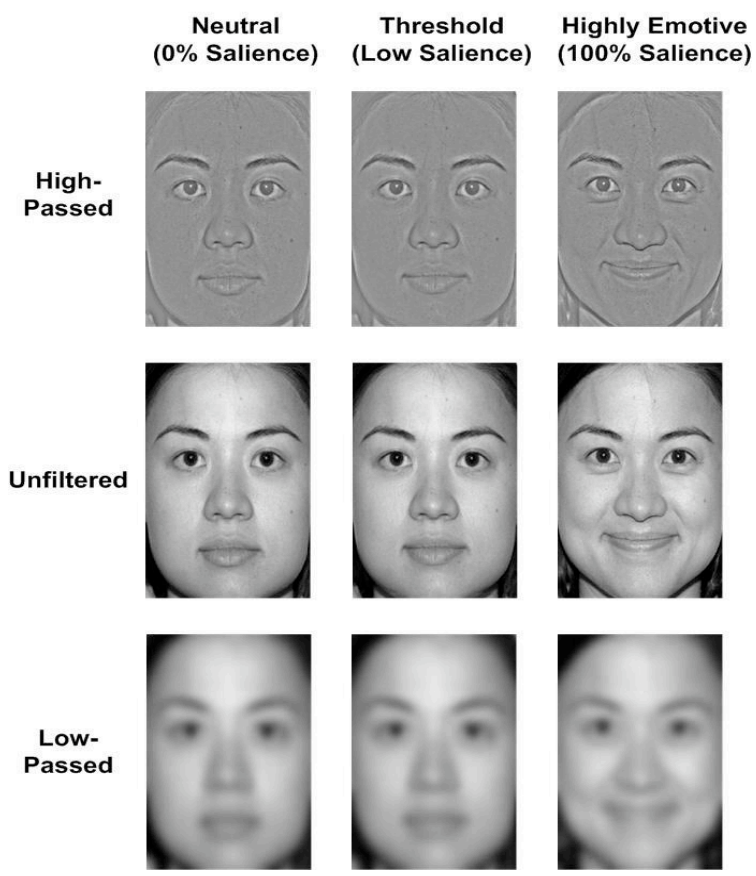

Fig. (6). Facial emotion images utilized for the happiness condition of the emotion perception task.

Together, these findings show a complex pattern that is not easily accounted for by current theories such as the low SF hypothesis put forward by Livingstone [18]. Livingstone theorized that LSF information facilitates individuals' perception that the Mona Lisa painting is smiling. The results of our emotion detection experiment are consistent with the hypothesis in that LSF information facilitates the detection of happiness. Our emotion perception experiment, on the other hand, yielded a different result: Happiness was more readily perceived in HSF images, which lacked LSF information. Note that the detection experiment involved one SF manipulation at a time, whereas the perception experiment required a direct comparison between two very different SF bands for which separate visual processing channels may be accessed. In light of this, our findings suggest 
that the role of LSFs in processing happiness information may depend on how visual processing channels are involved. When information from two visual processing channels is compared, the one utilized for HSF processing may be prioritized. When only one visual processing channel is stimulated, LSFs appear to be critical.

\subsection{High vs. Low Spatial Frequency Information}

On the emotion detection task, individuals' ability to detect happiness relied more on LSF than HSF information. This finding stands in contrast to a previous study [19], which found that facial expressions with low-peak frequencies were generally harder to detect than those defined by high-peak frequencies. A major difference in the previous study was that facial drawings were utilized, as opposed to face photographs (as used in our study). Line-drawings themselves do not contain much LSF information except for the configurations created by lines; it is therefore likely that in this earlier study there was a paucity of LSF information which could be utilized for emotion detection.

On the emotion perception task, HSF and LSF information worked in opposite directions when viewing the happiness stimuli: Images with HSFs were perceived as happier than those without HSFs, and images with LSFs were perceived as less happy than those without LSFs. Putting these results into perspective alongside the detection task, it may seem unusual that subjects were better at detecting happiness in LSF versus HSF images, but subjectively perceived images with HSF information as happier. In addition to the one vs. two visual processing channel hypothesis offered earlier, it may be the case that detection of happiness relies more heavily on a subcortical, lower-level face processing route which depends primarily on LSFs [20]. In contrast, emotion perception may require a more conscious, detailed analysis which involves the ventral visual cortex and HSF information [12].

Our finding that fear perception was not significantly affected by SF manipulations differs from the result of a previous study by Vuilleumier and colleagues [12], which showed that subjects perceived images with HSFs as more fearful than images without this information. One possible explanation is that this earlier study asked subjects to evaluate the fearfulness of individual images on a 5-point scale, whereas the present study employed direct comparisons of images containing different spatial frequency contents. It remains to be determined whether these methodological differences contribute to this discrepancy.

\subsection{Relationship between Emotional Salience and SF Information}

Modulation in SF information had a significant effect on individuals' perception of happiness at low levels of emotional salience $(0 \%$ and threshold levels), but not at high emotional salience $(100 \%)$. One possible explanation of this finding is that, at the $100 \%$ level, the emotional content of the images is so salient that the information available from either the LSF or HSF channel is sufficient for perception. Another possibility is that different perceptual mechanisms may be involved when viewing low and high salience emotions: At low salience, global features (LSFs) may play a more critical role in the perception of emotional content, whereas processing highly salient emotions may similarly depend upon localized (HSFs) and global (LSFs) features. While both interpretations highlight the contributions of visual features via a bottom-up process, the common thread suggests an adaptive association between the visual and affective systems which allows effective processing of facial emotion information. This suggestion has important implications on visual, cognitive and affective processing of face information in schizophrenia [21-23].

\subsection{Concluding Remarks}

One limitation of this study is that, in order to reduce performance fatigue, only one facial identity was used to systematically assess the role of spatial frequency information in emotion processing. This facial identity produced reliable and consistent results in a previous study [23]; however, our results should be further validated through use of additional facial identities in future studies.

In summary, this study presents novel data on the contributions of HSF and LSF information to two social cognitive processes: emotion detection and emotion perception. We found that SF-specific information has a particularly robust influence on individuals' recognition of happiness as opposed to fear, and at low salience as opposed to high salience. Future studies should extend this line of research by simultaneously investigating the effects of bottom-up (e.g., SF content) and top-down (e.g., selective attention) processes on individuals' abilities to recognize and interpret emotions. Additionally, researchers might examine the way in which perception of emotional features modulates basic visual perception [24], as this may yield complementary information to that reported in this study.

\section{CONFLICT OF INTEREST}

None declared.

\section{ACKNOWLEDGEMENTS}

This study was supported in part by a grant from the NIH (R01 MH 61824).

\section{REFERENCES}

[1] Haxby JV, Hoffman EA, Gobbini MI. Human neural systems for face recognition and social communication. Biol Psychiatry 2000;51(1): 59-67.

[2] Ishai A, Pessoa L, Bikle PC, Ungerleider LG. Repetition suppression of faces is modulated by emotion. Proc Natl Acad Sci USA 2004; 101(26): 9827-32.

[3] Pessoa L, Padmala S, Morland T. Fate of unattended fearful faces in the amygdala is determined by both attentional resources and cognitive modulation. Neuroimage 2005; 28(1): 249-55.

[4] Williams MA, McGlone F, Abbott DF, Mattingley JB. Differential amygdala responses to happy and fearful facial expressions depend on selective attention. Neuroimage 2005; 24(2): 417-25.

[5] Vuilleumier P, Armony JL, Driver J, Dolan RJ. Effects of attention and emotion on face processing in the human brain: an eventrelated fMRI study. Neuron 2001; 30(3): 829-41.

[6] De Valois R, De Valois K. Spatial Vision. New York: Oxford University Press; 1988.

[7] Fiorentini A, Maffei L, Sandini G. The role of high spatia frequencies in face perception. Perception 1983; 12(2):195-201. 
[8] Schyns PG, Oliva A. Dr. Angry and Mr. Smile: when categorization flexibly modifies the perception of faces in rapid visual presentations. Cognition 1999; 69(3): 243-65.

[9] Iidaka T, Yamashita K, Kashikura K, Yonekura Y. Spatial frequency of visual image modulates neural responses in the temporo-occipital lobe. An investigation with event-related fMRI. Brain Res Cogn Brain Res 2004; 18(2):196-204.

[10] Rotshtein P, Vuilleumier P, Winston J, Driver J, Dolan R. Distinct and convergent visual processing of high and low spatial frequency information in faces. Cereb Cortex 2007; 17(11): 2713-24.

[11] Alorda C, Serrano-Pedraza I, Campos-Bueno JJ, Sierra-Vazquez V, Montoya P. Low spatial frequency filtering modulates early brain processing of affective complex pictures. Neuropsychologia 2007; 45(14): 3223-33

[12] Vuilleumier P, Armony JL, Driver J, Dolan RJ. Distinct spatial frequency sensitivities for processing faces and emotional expressions. Nat Neurosci 2003; 6(6): 624-31.

[13] White M, Li J. Matching faces and expressions in pixelated and blurred photos. Am J Psychol 2006;119(1): 21-8.

[14] Wechsler D. Manual for the Adult Intelligence Scale-Revised. New York: Psychological Corporation; 1981.

[15] First MB, Spitzer RL, Gibbon M, William JB. Structure Clinical Interview for DSM -IV-TR Axis I Disorders - Non-patient ed (SCID-I/NP, 11/2002 revision) New York, NY Biometric Research Department, New York State Psychiatric Institute; 2002.
[16] Tottenham N, Tanaka JW, Leon AC, et al. The NimStim set of facial expressions: judgments from untrained research participants. Psychiatry Res 2009; 168(3): 242-9.

[17] Smith ML, Cottrell GW, Gosselin F, Schyns PG. Transmitting and decoding facial expressions. Psychol Sci 2000;16(3):184-9.

[18] Livingstone MS. Is it warm? Is it real? Or just low spatial frequency? Science 2000; 290(5495): 1299.

[19] Goren D, Wilson HR. Quantifying facial expression recognition across viewing conditions. Vision Res 2006; 46(8-9):1253-62.

[20] Johnson MH. Subcortical face processing. Nat Rev Neurosci 2005; 6(10):766-74.

[21] Chen Y, Norton D, McBain R, Ongur D, Heckers S. Visual and cognitive processing of face information in schizophrenia: detection, discrimination and working memory. Schizophr Res 2009;107(1): 92-8.

[22] McBain R, Norton D, Chen Y. Differential roles of low and high spatial frequency content in abnormal facial emotion perception in schizophrenia. Schizophr Res 2011;122(1-3): 151-5.

[23] Norton D, McBain R, Holt DJ, Ongur D, Chen Y. Association of impaired facial affect recognition with basic facial and visual processing deficits in schizophrenia. Biol Psychiatry 2009; 65(12): 1094-8.

[24] Bocanegra BR, Zeelenberg R. Emotion improves and impairs early vision. Psychol Sci 2009; 20(6): 707-13.

(C) McBain et al.; Licensee Bentham Open.

This is an open access article licensed under the terms of the Creative Commons Attribution Non-Commercial License (http://creativecommons.org/licenses/by$\mathrm{nc} / 3.0 /$ ), which permits unrestricted, non-commercial use, distribution and reproduction in any medium, provided the work is properly cited. 